\title{
High-density lipoproteins: a novel therapeutic target for cardiovascular disease
}

REVIEW

This article was published in the following Dove Press journal:

Research Reports in Clinical Cardiology

I2 January 201 I

Number of times this article has been viewed

\author{
TS Mohamed Saleem' \\ PV Sandhya Rani' \\ K Gauthaman ${ }^{2}$ \\ 'Department of Pharmacology, \\ Annamacharya College of Pharmacy, \\ New Boyanapalli, Andhrapradesh, \\ India; ${ }^{2}$ Department of Drug \\ Technology, Faculty of Medical \\ Technology, Derna, Libya
}

\begin{abstract}
Cardiovascular disease has a high rate of mortality in both Western and developing countries. Atherosclerosis and generation of reactive oxygen species through oxidative stress is the major risk factor for cardiovascular disease. Atherothrombosis with low levels of high-density lipoprotein (HDL) and high levels of low-density lipoprotein is a major risk factor for atherosclerosis-induced cardiovascular disease. Lipid-lowering drugs like statins, niacin, fibrates, and some newer agents, ie, the apolipoprotein A-I mimetics and the cholesteryl ester transfer protein inhibitors, not only increase HDL levels but are also effective in reducing key atherogenic lipid components, including triglyceride-rich lipoproteins. The aim of this review is to discuss the accumulating evidence suggesting that HDL possesses a diverse range of biological actions, and that increasing HDL levels by drug treatment may be beneficial in the prevention of cardiovascular disease.
\end{abstract}

Keywords: cardiovascular disease, lipoproteins, statins, apolipoprotein, atherosclerosis

\section{Introduction}

Atherothrombosis is a disease caused by low levels of high-density lipoprotein (HDL) and elevated levels of low-density lipoprotein (LDL). A low plasma concentration of HDL is an important risk factor for atherosclerosis-induced cardiovascular disease. ${ }^{1,2}$ Epidemiological studies have shown that low HDL is an independent risk factor for cardiovascular disease associated with diabetes mellitus and metabolic syndrome, even in patients having normal LDL levels. ${ }^{3,4}$ This report highlights some of the therapeutic implications of the National Cholesterol Education Program - Adult Treatment Panel III guidelines report and various therapeutic approaches to lowering elevated LDL and triglycerides, as well as increasing low levels of HDL to optimize clinical event rate reduction in patients with coronary heart disease. ${ }^{5} \mathrm{New}$ insights into the basic science of HDL function and metabolism, such as the discovery of beta-chain adenosine triphosphate (ATP) synthase as a hepatic catabolic HDL receptor, are further characterizing the importance of HDL in atheroprotection and identifying novel targets of drug development. Nicotinic acid, fibrates, statins, and thiazolidinediones not only increase HDL levels but also alter the size and composition of HDL subpopulations. Furthermore, these drugs promote the direct antiatherogenic effects of HDL, ie, antioxidation, anti-inflammatory and antithrombotic effects, and endothelial stabilization. In addition, there has been an increasing awareness in recent years of the importance of triglyceride-rich lipoprotein as a risk factor for cardiovascular disease, and the advantages of reducing plasma triglyceride levels and raising HDL levels to prevent coronary events. ${ }^{6}$
Correspondence: TS Mohamed Saleem Department of Pharmacology, Annamacharya College of Pharmacy, New Boyanapalli, Rajampet-516/26, Andhrapradesh, India

Tel $+9 \mid 9701978543$

$\mathrm{Fax}+919542326252$

Email saleemcology@gmail.com
Research Reports in Clinical Cardiology 201 I:2 I-6

(c) 20II Saleem et al, publisher and licensee Dove Medical Press Ltd.This is an Open Access article which permits unrestricted noncommercial use, provided the original work is properly cited.
Dovepress

DOI: 10.2147/RRCC.S1 5600 
Thus, therapeutic intervention aimed at raising HDL, within the context of reducing global cardiovascular risk, would benefit patients, and is a viewpoint increasingly adopted by international treatment guidelines. Therapeutic options for patients with low HDL include statins, fibrates, and nicotinic acid, either as monotherapy or in combination. Of these options, nicotinic acid is not only the most potent agent for raising HDL, but is also effective in reducing key atherogenic lipid components, including triglyceride-rich lipoproteins. ${ }^{7}$

\section{HDL and cardiovascular disease}

Individuals with low HDL cholesterol levels, ie, $<40 \mathrm{mg} / \mathrm{dL}$ $(1.03 \mathrm{mmol} / \mathrm{L})$ in men and $<50 \mathrm{mg} / \mathrm{dL}(1.30 \mathrm{mmol} / \mathrm{L})$ in women, are at increased risk of coronary heart disease, restenosis after angioplasty, ${ }^{8}$ and death from cardiovascular causes, especially if male ${ }^{9}$ or with diabetes. ${ }^{10}$ People with low HDL levels also have other cardiovascular risk factors, such as diabetes, hypertension, or both, which further increase risk. Epidemiologic and animal studies suggest that raising HDL levels may delay the development of atherosclerosis. In humans, each increase in baseline HDL of $1 \mathrm{mg} / \mathrm{dL}(0.03 \mathrm{mmol} / \mathrm{L})$ is associated with a $6 \%$ decrease in the risk of death from coronary disease or myocardial infarction. ${ }^{11}$ Another study suggests that for every $1 \mathrm{mg} / \mathrm{dL}$ $(0.03 \mathrm{mmol} / \mathrm{L})$ increase in HDL, cardiovascular risk is reduced by $2 \%-3 \%{ }^{4}$

\section{HDL as a novel therapeutic target Statins}

When lipid-lowering therapy is necessary, statins are preferred over other lipid-lowering drugs because they not only target dyslipidemia but also have anti-inflammatory properties. Statin subgroup analysis reveals no important differences between the three most commonly prescribed statins, ie, simvastatin, atorvastatin, and pravastatin. Atorvastatin produced greater reductions in LDL and triglyceride levels than other statins at milligram-equivalent doses, but had a limited ability to increase HDL levels. Indeed, increasing doses of atorvastatin were associated with progressively smaller increases in HDL levels, and a dose of $80 \mathrm{mg}$ was associated with a mean decrease of $0.1 \%$ from baseline HDL level and, similarly, fluvastatin $40 \mathrm{mg}$ lowered HDL levels by $3 \%{ }^{12}$

The consequences of LDL and other lipoproteins against cardiovascular disease have already been reported by physicians. This is the main reason for the therapeutic need to decrease cardiovascular risk using lipid-lowering drugs. Recent studies show that statins demonstrate efficacy against this disease not only by reducing LDL levels but also by increasing HDL levels. ${ }^{13}$ Many recent trials have demonstrated the effects of statins in cardiovascular disease, particularly reducing LDL and increasing HDL, and these effects have not been identified in patients with diabetes. ${ }^{14}$ The most recent National Cholesterol Education Program - Adult Treatment Panel III guidelines suggest that statin therapy reduces cardiovascular disease risk by decreasing LDL levels and other mechanisms, including anti-inflammatory actions, and alteration of HDL and apolipoprotein levels. ${ }^{2}$

Large-scale, randomized, prospective trials involving patients with coronary heart disease have shown that statins reduce the clinical consequences of atherosclerosis, including cardiovascular deaths, nonfatal myocardial infarction and stroke, hospitalization for acute coronary syndrome and heart failure, as well as the need for coronary revascularization. ${ }^{15}$ Although the clinical benefits of statins have been well documented by primary and secondary prevention trials that justify their widespread use, it was not until the publication of the Veterans Affairs High-Density Lipoprotein Intervention Trial (VA-HIT) that the relevance of identifying HDL as a therapeutic target to reduce the risk of recurrent coronary heart disease events was identified. ${ }^{16}$

Statins, particularly atorvastatin, reduce triglyceride-rich lipoprotein levels and increase HDL more than other lipidlowering drugs, eg, fenofibrate, and have a greater impact on atherogenic risk ratios, such as LDL:HDL. ${ }^{17}$ In addition to modifying the lipid profile, statins have beneficial cholesterol-independent pleiotropic effects. The contribution of these effects to event reduction is not yet fully understood. ${ }^{6}$ Statins, in addition to lowering LDL levels, raise HDL levels by $2 \%-15 \%$ via increased apolipoprotein A-1 synthesis. ${ }^{18}$

HATS (the HDL Atherosclerosis Treatment Study) ${ }^{19}$ demonstrated that a combination of low-dose simvastatin (10-20 mg/day) and high-dose niacin (2-4 mg/day) significantly increased HDL levels by $26 \%$, as compared with placebo in patients with HDL levels $\leq 40 \mathrm{mg} / \mathrm{L}$, LDL levels $\leq 145 \mathrm{mg} / \mathrm{dL}(3.75 \mathrm{mmol} / \mathrm{L})$, and triglyceride levels $<400 \mathrm{mg} / \mathrm{dL}(4.52 \mathrm{mmol} / \mathrm{L})$. In the Arterial Biology for the Investigation of the Treatment Effects of Reducing Cholesterol 2 (ARBITER 2) study, ${ }^{20}$ which included subjects with established coronary disease and HDL $<45 \mathrm{mg} / \mathrm{dL}$ $(1.16 \mathrm{mmol} / \mathrm{L})$ and LDL cholesterol levels $<130 \mathrm{mg} / \mathrm{dL}$ (3.36 mmol/L), showed that addition of extended-release niacin $1000 \mathrm{mg}$ daily to existing statin therapy increased mean HDL levels by $21 \%$ (from a mean $39-47 \mathrm{mg} / \mathrm{dL}$ [1.01-1.21 mmol/L]; $P<0.001$ versus the change in the placebo group). Medial thickness of the carotid intima 
significantly increased in the placebo group (mean change, $0.044 \mathrm{~mm} ; P<0.001$ ) but not in the niacin group (mean change, $0.014 \mathrm{~mm} ; P=0.23$ ). However, a comparison of changes in intimal thickness over time did not show a significant difference between the two treatment groups. ${ }^{18}$

\section{Nicotinic acid}

Niacin (nicotinic acid or vitamin B) is the most effective medication to raise HDL cholesterol levels, causing increases of 20\%-35\%. The Coronary Drug Project demonstrated a significant reduction in the incidence of death and myocardial infarction after five years of niacin treatment in men with a history of myocardial infarction. Niacin inhibits hepatic uptake of apolipoprotein A-I and increases plasma pre- $\beta$ HDL levels. Niacin therapy is associated with improved endothelial function and nitric oxide synthase activity. ${ }^{18}$ In another study, Carlson reviewed the use of nicotinic acid along with statins in atherosclerosis-induced cardiovascular disease, and found that nicotinic acid increased HDL levels and decreased LDL levels in these patients. ${ }^{1}$ However, despite statin therapy, a significant number of cardiovascular events continues to occur, indicating the need for additional targets for atherosclerosis management.

\section{Bile acid sequestrants}

The two established bile acid sequestrants or resins, cholestyramine and colestipol, are among the oldest of the hypolipidemic drugs, and are probably the safest agents because they are not absorbed from the intestine. These resins are also recommended for patients aged 11-20 years. Because statins are effective as monotherapy, resins are most often used as second-line agents if statin therapy does not lower LDL levels sufficiently. When used with a statin, cholestyramine and colestipol are usually prescribed at submaximal doses. Inhibition of reductase activity by a statin substantially increases the effectiveness of the resins. Maximal doses (24 $\mathrm{g}$ of cholestyramine and $30 \mathrm{~g}$ of colestipol) may reduce LDL by up to $25 \%$, but will cause gastrointestinal side effects that are poorly tolerated by most patients. One to two weeks is sufficient to attain maximal LDL reduction using a given resin dose. HDL levels increase by $4 \%$ to $5 \%$. Statins plus resins or niacin plus resins can reduce LDL by up to $40 \%-60 \%{ }^{21}$

\section{Fibric acid derivatives}

In another study it has been reported that obesity, the so-called metabolic syndrome, is a significant risk factor for coronary heart disease with high triglyceride levels and low HDL levels.
Fibrates have been used in clinical practice in more than two decoronary artery diseases and many clinical studies have reported that the use of fibrates in obese patients reduces the risk of cardiovascular disease by increasing HDL levels. ${ }^{16}$

Gemfibrozil is more effective than the statins in decreasing triglycerides and increasing HDL in diabetic patients, and may even increase LDL levels (fenofibrate may be an exception, decreasing LDL cholesterol by $20 \%-25 \%$ in some studies). However, gemfibrozil does increase LDL particle size. Nevertheless, the statins are the current lipid-lowering drugs of choice because the change in the LDL to HDL ratio is better than for gemfibrozil. Moreover, the diabetic patient may be more likely to benefit from statin therapy than the nondiabetic patient. ${ }^{22}$

In the Helsinki Heart Study and VA-HIT, treatment with gemfibrozil was associated with a reduction in cardiovascular disease events. The $22 \%$ reduction in coronary events in VA-HIT was attributed to a modest increase $(6 \%)$ in HDL levels. In contrast, the results of studies using other fibrates, such as bezafibrate and fenofibrate, have been negative, while clofibrate has been associated with potential hazards. Some evidence indicates that the benefits seen in VA-HIT may be partly due to the additive effects of fibrates on apolipoprotein synthesis and lipoprotein metabolism. The combination of fibrates, particularly gemfibrozil, with statins requires caution and monitoring of creatine kinase levels because of the risk of myotoxicity, including rhabdomyolysis. ${ }^{23}$

\section{Newer drugs Apolipoprotein A-I mimetics}

Recent reports indicate that apolipoprotein A-1 mimetics inhibit atherosclerosis in LDL receptor-null mice fed Western diets. ${ }^{24}$ The apolipoprotein A-I mimetic, 5A peptide, is highly specific for ATP-binding cassette transporter A1-mediated cholesterol efflux. Like apolipoprotein A-I, 5A peptide inhibits acute inflammation and oxidative stress in rabbit carotid vessels and human coronary artery endothelial cells. In vitro, the $5 \mathrm{~A}$ peptide exerts these beneficial effects through interaction with the ATP-binding cassette A1 transporter and nuclear factor- $\kappa \mathrm{B}(\mathrm{NF}-\kappa \mathrm{B})$ signaling pathways. ${ }^{25}$

Apolipoprotein A-1 mimetic peptides are potent antioxidant and anti-inflammatory compounds which are highly effective in ameliorating atherosclerosis and inflammation in experimental animals. Apolipoprotein $\mathrm{E}^{-/-}$mice develop proteinuria and glomerular and tubulointerstitial injury associated with upregulation of oxidative and inflammatory mediators in the kidney, which is ameliorated by the administration of apolipoprotein A-1 mimetic peptide. These observations 
point to the role of oxidative stress and inflammation in the pathogenesis of renal disease in hyperlipidemic animals and perhaps in humans. ${ }^{26} \mathrm{D}-4 \mathrm{~F}$ and pravastatin when given in combination at oral doses that were ineffective when given as single agents rendered HDL anti-inflammatory in mice and monkeys, prevented atherosclerosis in young apolipoprotein $\mathrm{E}$ null mice, and caused regression of established lesions in older ones. These results suggest that the combination of a statin and an HDL-based therapy may be a particularly potent treatment strategy. ${ }^{27}$ Metabolic syndrome and type 2 diabetes are characterized not only by elevated cardiovascular risk and by low HDL cholesterol levels, but also by defective HDL function. Deficient HDL function and subnormal HDL levels may act synergistically to accelerate atherosclerosis in metabolic disease. Therapeutic normalization of attenuated antiatherogenic HDL function in terms of both particle number and quality of HDL particles is the target of innovative pharmacological approaches to increasing HDL, including inhibition of cholesteryl ester transfer protein (CETP), enhanced lipidation of apolipoprotein A-I with nicotinic acid, and infusion of reconstituted HDL or apolipoprotein A-I mimetics. A preferential increase in circulating concentrations of HDL particles possessing normalized antiatherogenic activity is therefore a promising therapeutic strategy for the treatment of common metabolic diseases featuring dyslipidemia, inflammation, and premature atherosclerosis. $^{28}$

\section{Cholesteryl ester transfer protein inhibitors}

CETP is a plasma glycoprotein that mediates the transfer of cholesteryl esters from HDL to apolipoprotein B-containing lipoproteins, with a balanced transfer of triglycerides. Inhibition of CETP results in an accumulation of cholesteryl esters in HDL, thus resulting in increased HDL. ${ }^{29} \mathrm{CETP}$ inhibitors induce tight binding of CETP to HDL, indicating that these inhibitors promote the formation of a complex between CETP and HDL, resulting in inhibition of CETP activity. ${ }^{30}$

In a double-blind trial with participants randomized in a 2:1 ratio to dalcetrapib $900 \mathrm{mg}$ administered for up to 48 weeks, Stein et $\mathrm{al}^{31}$ demonstrated no clinically relevant changes in lymph nodes, blood pressure, or other safety parameters. In this trial, participants were randomized in a $2: 1$ ratio to dalcetrapib $900 \mathrm{mg} /$ day (higher than the $600 \mathrm{mg}$ Phase III dose) or placebo, both with atorvastatin, for 24 weeks. Dalcetrapib increased HDL (by 33.4\% at week 24 and 33.8\% at week 48), decreased CETP activity ( $-53.5 \%$ at week 24 and $-56.5 \%$ at week 48$)$, and increased apolipoprotein A-I (11.4\% at week 24 and $16.4 \%$ at week 48). Another study strongly supported the concept that anacetrapib seems to exhibit HDL increases greater than those seen with other investigational drugs in this class and demonstrates LDL-lowering effects similar to those of statins and anacetrapib without increasing blood pressure. ${ }^{32}$

The CETP inhibitor torcetrapib increases plasma HDL levels by $40 \%-60 \%$, while modestly decreasing LDL levels. Combining the HDL-elevating properties of a CETP inhibitor with the LDL cholesterol-lowering properties of a statin may offer improved outcomes over targeting LDL alone. ${ }^{33}$

Torcetrapib has been shown to decrease aortic atherosclerosis in animals and significantly increase HDL levels in humans. ${ }^{34-36}$ However, development of torcetrapib was halted in response to the results of Investigation of Lipid Level management to Understand its iMpact IN ATherosclerosis Events (ILLUMINATE), which showed a 61\% increase in all-cause mortality and other cardiovascular events in individuals at high risk for congestive heart disease who were treated with torcetrapib plus atorvastatin compared with those who received placebo plus atorvastatin. ${ }^{23}$

\section{Ongoing research}

The beneficial effects of HDL on the cardiovascular system have been attributed to its ability to remove cellular cholesterol, as well as its anti-inflammatory, antioxidant, and antithrombotic properties, which act in concert to improve endothelial function and inhibit atherosclerosis, thereby reducing cardiovascular risk. ${ }^{4}$

Emerging HDL-raising therapies (such as CTEP inhibitors and 1,2-dimyristoyl-sn-glycero-phosphocholine) and novel interventions that mimic the beneficial effects of HDL (such as apolipoprotein A-I milano and apolipoprotein A-I mimetic peptides) are proving beneficial in animal and human studies. ${ }^{37}$ A number of preclinical studies have suggested that several HDL-based therapies have the potential to stabilize or regress atherosclerosis, consistent with epidemiologic evidence of an inverse relationship between coronary heart disease and HDL cholesterol levels. One such therapeutic approach involves direct infusion of HDL or HDL-like molecules for rapid remodeling and stabilization of atherosclerosis. ${ }^{38}$

A variety of agents are being developed which affect both LDL and HDL metabolism, including inhibitors of acylcoenzyme A-cholesterol acyl transferase, microsomal transfer protein, and CETP, as well as specific receptor agonists. Growing research suggests that increasing HDL levels is important to limit cardiovascular risk in many patients. In addition, the pleiotropic anti-inflammatory effects of lipidlowering therapy may provide further risk reduction. ${ }^{39}$ 
Some of the treatments that increase HDL concentrations have been shown to reduce atheroma volume and may prevent the formation of new atherosclerotic lesions, thereby reducing the risk of cardiovascular disease. For these reasons, increasing HDL concentrations has become a potentially attractive therapeutic target for individuals who are at increased risk of cardiovascular disease, including those with metabolic syndrome. Traditional strategies to increase HDL include the use of niacin, statins, and fibric acid derivatives. Pharmacotherapies that have recently been developed and are currently being evaluated include inhibition of CETP and antagonism of the endocannabinoid CB1 receptor. Initial studies of CETP inhibitors suggest that these agents may markedly increase HDL concentrations. Clinical trials with rimonabant, a CB1 receptor antagonist, have demonstrated significant weight loss, as well as increased HDL levels and reduced triglyceride levels. ${ }^{40}$

In many trials, it has been reported that currently available therapeutic and lifestyle strategies, when optimized, increase HDL levels by $20 \%-30 \%$. While basic and small pilot studies have shown promise, proof that increasing HDL levels confers a reduction in major cardiovascular outcomes independent of changes in levels of LDL cholesterol or triglycerides has been more elusive. Some novel therapeutic agents in human studies appear to increase HDL levels effectively, whereas other novel strategies that target HDL metabolism or function may have minimal effect on HDL levels. ${ }^{39}$ Statins have been shown to decrease LDL levels, but the evidence for reduction of coronary artery disease risk with HDL-raising therapy is relatively thin, partly due to the scarcity of effective and safe drugs for increasing HDL levels. ${ }^{3}$

\section{Conclusion}

Development of new pharmaceutical agents for raising HDL represents an important strategy for reducing the residual cardiovascular risk associated with atherothrombosis.

\section{Disclosure}

The authors declare no conflicts of interest in this work.

\section{References}

1. Carlson LA. Nicotinic acid and other therapies for raising high-density lipoprotein. Curr Opin Cardiol. 2006;21(4):336-344.

2. Evans M, Roberts A, Davies S, Rees A. Medical lipid-regulating therapy: Current evidence, ongoing trials and future developments. Drugs. 2004;64(11):1181-1196.

3. Venkatesh PK, Caskey D, Reddy PC. Therapies to increase high-density lipoprotein cholesterol and their effect on cardiovascular outcomes and regression of atherosclerosis. Am J Med Sci. 2008;336(1):64-68.

4. Hausenloy DJ, Yellon DM. Targeting residual cardiovascular risk: Raising high-density lipoprotein cholesterol levels. Postgrad Med J. 2008;84(997):590-598.
5. Boden WE. Therapeutic implications of recent ATP III guidelines and the important role of combination therapy in total dyslipidemia management. Curr Opin Cardiol. 2003;18(4):278-285.

6. Fabbri G, Maggioni AP. Cardiovascular risk reduction: What do recent trials with rosuvastatin tell us? Adv Ther. 2009;26(5):469-487.

7. Chapman MJ, Assmann G, Fruchart JC, Shepherd J, Sirtori C; European Consensus Panel on HDL-C. Raising high-density lipoprotein cholesterol with reduction of cardiovascular risk: The role of nicotinic acid - a position paper developed by the European Consensus Panel on HDL-C. Curr Med Res Opin. 2004;20(8):1253-1268.

8. Shah PK, Kaul S, Nilsson J, Cercek B. Exploiting the vascular protective effects of high-density lipoprotein and its apolipoproteins: An idea whose time for testing is coming, part I. Circulation. 2001;104(19): 2376-2383.

9. Wilson PW, Abbott RD, Castelli WP. High density lipoprotein cholesterol and mortality: The Framingham Heart Study. Arteriosclerosis. 1988;8(6):737-741.

10. Goldbourt U, Yaari S, Medalie JH. Isolated low HDL cholesterol as a risk factor for coronary heart disease mortality: A 21-year follow-up of 8000 men. Arterioscler Thromb Vasc Biol. 1997;17(1):107-113.

11. Gordon DJ, Knoke J, Probstfield JL, Superko R, Tyroler HA. High-density lipoprotein cholesterol and coronary heart disease in hypercholesterolemic men: The Lipid Research Clinics Coronary Primary Prevention Trial. Circulation. 1986;74(6):1217-1225.

12. Nurmohamed MT, Dijkmans BA. Dyslipidaemia, statins and rheumatoid arthritis. Ann Rheum Dis. 2009;68(4):453-455.

13. Badimon JJ, Ibanez B. Increasing high-density lipoprotein as a therapeutic target in atherothrombotic disease. Rev Esp Cardiol. 2010; 63(3):323-333. Spanish.

14. Cziraky MJ, Watson KE, Talbert RL. Targeting low HDL-cholesterol to decrease residual cardiovascular risk in the managed care setting. J Manag Care Pharm. 2008;14(8 Suppl):S3-S28.

15. Tenenbaum A, Fisman EZ, Motro M, Adler Y. Optimal management of combined dyslipidemia: What have we behind statins monotherapy? Adv Cardiol. 2008;45:127-153.

16. Després JP, Lemieux I, Robins SJ. Role of fibric acid derivatives in the management of risk factors for coronary heart disease. Drugs. 2004; 64(19):2177-2198.

17. Davignon J. Advances in drug treatment of dyslipidemia: Focus on atorvastatin. Can J Cardiol. 1998;14 Suppl B:28B-38B.

18. Ashen MD, Blumenthal R. Low HDL cholesterol levels. N Engl J Med. 2005;353(12):1252-1260.

19. Brown BG, Zhao X-Q, Chait A, et al. Simvastatin and niacin, antioxidant vitamins, or the combination for the prevention of coronary disease. N Engl J Med. 2001;345(22):1583-1592.

20. Taylor AJ, Sullenberger LE, Lee HJ, Lee JK, Grace KA. Arterial Biology for the Investigation of the Treatment Effects of Reducing Cholesterol (ARBITER) 2: A double-blind, placebo-controlled study of extendedrelease niacin on atherosclerosis progression in secondary prevention patients treated with statins. Circulation. 2004;110(23):3512-3517.

21. Mahley RW, Bersot TP. Drug therapy for hypercholesterolemia and dyslipidemia. In: Brunton LL, Lazo SJ, Parker KL, editors. Goodman and Gilman's The Pharmacological Basis of Therapeutics. 11th ed. New York, NY: McGraw Hill; 2006.

22. Kreisberg RA. Diabetic dyslipidemia. Am J Cardiol. 1998;82(12A): 67U-73U.

23. Inder MS, Shishehbor MH, Ansell BJ. High density lipoprotein as a therapeutic target. JAMA. 2007;298(7):786-798.

24. Ou J, Ou Z, Jones DW, et al. L-4F, an apolipoprotein A-1 mimetic, dramatically improves vasodilation in hypercholesterolemia and sickle cell disease. Circulation. 2003;107(18):2337-2341.

25. Tabet F, Remaley AT, Segaliny AI, et al. The 5A apolipoprotein A-I mimetic peptide displays antiinflammatory and antioxidant properties in vivo and in vitro. Arterioscler Thromb Vasc Biol. 2010;30(2):246-252.

26. Vaziri ND, Kim HJ, Moradi H, et al. Amelioration of nephropathy with apoA-1 mimetic peptide in apo-E deficient mice. Nephrol Dial Transplant. 2010;25(11):3525-3534. 
27. Navab M, Anantharamaiah GM, Hama S, et al. D-4F and statins synergize to render HDL antiinflammatory in mice and monkeys and cause lesion regression in old apolipoprotein E-null mice. Arterioscler Thromb Vasc Biol. 2005;25(7):1426-1432.

28. Kontush A, Chapman MJ. Functionally defective high-density lipoprotein: A new therapeutic target at the crossroads of dyslipidemia, inflammation, and atherosclerosis. Pharmacol Rev. 2006;58(3):342-374.

29. Hunt JA, Lu Z. Cholesteryl ester transfer protein (CETP) inhibitors. Curr Top Med Chem. 2009;9(5):419-427.

30. Ranalletta M, Bierilo KK, Chen Y, et al. Biochemical characterization of cholesteryl ester transfer protein inhibitors. J Lipid Res. 2010;51(9): 2739-2752.

31. Stein EA, Roth EM, Rhyne JM, Burgess T, Kallend D, Robinson JG. Safety and tolerability of dalcetrapib (RO4607381/JTT-705): Results from a 48-week trial. Eur Heart J. 2010;31(4):480-488.

32. Krishna R, Anderson MS, Bergman AJ, et al. Effect of the cholesteryl ester transfer protein inhibitor, anacetrapib, on lipoproteins in patients with dyslipidaemia and on 24-h ambulatory blood pressure in healthy individuals: Two double-blind, randomised placebo-controlled phase I studies. Lancet. 2007;370(9603):1907-1914.

33. Tardif JC. Prevention challenges: The era of atherosclerosis regression. Can J Cardiol. 2006;22 Suppl C:27C-30C.
34. Brousseau ME, Schaefer EJ, Wolfe ML, et al. Effects of an inhibitor of cholesteryl ester transfer protein on HDL cholesterol. $N$ Engl J Med. 2004;350(15):1505-1515.

35. Clark RW, Sutfin TA, Ruggeri RB, et al. Raising high-density lipoprotein in humans through inhibition of cholesteryl ester transfer protein: An initial multidose study of torcetrapib. Arterioscler Thromb Vasc Biol. 2004;24(3):490-497.

36. Morehouse LA, Sugarman ED, Bourassa PA, et al. Inhibition of CETP activity by torcetrapib reduces susceptibility to diet-induced atherosclerosis in New Zealand white rabbits. J Lipid Res. 2007;48(6): 1263-1272.

37. Meyers CD, Kashyap ML. Pharmacologic elevation of high-density lipoproteins: Recent insights on mechanism of action and atherosclerosis protection. Curr Opin Cardiol. 2004;19(4):366-373.

38. Shah PK. Apolipoprotein A-I/HDL infusion therapy for plaque stabilization-regression: A novel therapeutic approach. Curr Pharm Des. 2007;13(10):1031-1038.

39. Singh IM, Shishehbor MH, Ansell BJ. High-density lipoprotein as a therapeutic target: A systematic review. JAMA. 2007;298(7):786-798.

40. Cannon CP. High-density lipoprotein cholesterol and residual cardiometabolic risk in metabolic syndrome. Clin Cornerstone. 2007; 8 Suppl 6:S14-S23.
Research Reports in Clinical Cardiology

\section{Publish your work in this journal}

Research Reports in Clinical Cardiology is an international, peerreviewed, open access journal publishing original research, reports, editorials, reviews and commentaries on all areas of cardiology in the clinic and laboratory. The manuscript management system is completely online and includes a very quick and fair peer-review system. Visit

\section{Dovepress}

http://www.dovepress.com/testimonials.php to read real quotes from published authors. 